\title{
INTEGRAL CHARACTERIZATIONS FOR STABILITY OF LINEAR SKEW-PRODUCT SEMIFLOWS
}

\author{
ADINA LUMINIŢA SASU
}

Abstract. We give necessary and sufficient conditions for uniform exponential stability of linear skew-product semiflows. Using the theory of Banach function spaces we obtain integral characterizations for this concept. We extend a stability theorem obtained by Rolewicz for evolution families, at the general case of linear skew-product semiflows.

Mathematics subject classification (2000): 34D05, 46E30, 93D20. semiflows.

Key words and phrases: uniform exponential stability, Banach function spaces, linear skew product

\section{REFERENCES}

[1] C. Chicone, Y. LATUshrin, Evolution Semigroups in Dynamical Systems and Differential Equations, Math. Surveys and Monographs, vol. 70, Amer. Math. Soc., 1999.

[2] S. N. CHOw, H. LeIVA, Dynamical spectrum for time-dependent linear systems in Banach spaces, Japan J. Indust. Appl. Math. 11 (1994), 379-415.

[3] S. N. CHOW, H. LEIVA, Existence and roughness of the exponential dichotomy for linear skew-product semiflow in Banach space, J. Differential Equations 102 (1995), 429-477.

[4] Y. LATUShKin, S. MONTGOMERY-SMith, T. RANDOLPH, Evolutionary semigroups and dichotomy of linear skew-product flows on locally compact spaces with Banach fibres, J. Differential Equations 125 (1996), 75-116.

[5] Y. LATUSHKIn, R. SCHNAUBELT, Evolution semigroups, translation algebras and exponential dichotomy of cocycles, J. Differential Equations 159 (1999), 321-369.

[6] R. MANÁSEVICH, J. MAWHIN, The spectrum of p-Laplacian systems with various boundary conditions and applications, Adv. Differential Equations 5 (2000), 1289-1318.

[7] J. Mawhin, Analyse. Fondements, techniques, évolution, De Boeck Université, Belgium (1997).

[8] J. MAWHIN, J. R. WARD, Guiding-like functions for periodic or bounded solutions of ordinary differential equations, Discrete Contin. Dynam. Systems 8 (2002), 39-54.

[9] M. Megan, B. SASU, A. L. SASU, On uniform exponential stability of evolution families, Riv. Mat. Univ. Parma 4 (2001), 27-43.

[10] M. Megan, A. L. Sasu, B. Sasu, A. Pogan, Exponential stability and instability of semigroups of linear operators in Banach spaces, Math. Inequal. Appl. 5 (2002), 557-568.

[11] M. Megan, A. L. SASU, B. SASU, On uniform exponential stability of linear skew-product semiflows in Banach spaces, Bull. Belg. Math. Soc. - Simon Stevin 9 (2002), 143-154.

[12] M. Megan, B. SASU, A. L. SASu, On nonuniform exponential dichotomy of evolution operators in Banach spaces, Integral Equations Operator Theory 44 (2002), 71-78.

[13] M. Megan, A. L. Sasu, B. Sasu, Stabilizability and controllability of systems associated to linear skew-product semiflows, Rev. Mat. Complutense 15 (2002), 599-618.

[14] M. Megan, A. L. SAsu, B. SASu, Discrete admissibility and exponential dichotomy for evolution families, Discrete Contin. Dynam. Systems 9 (2003), 383-397.

[15] M. MEgan, A. L. SASU, B. SASU, Theorems of Perron type for uniform exponential dichotomy of linear skew-product semiflows, Bull. Belg. Math. Soc. - Simon Stevin 10 (2003), 1-21. 
[16] M. Megan, A. L. Sasu, B. SASu, Perron conditions for uniform exponential expansiveness of linear skew-product flows, Monatsh. Math. 138 (2003), 145-157.

[17] M. Megan, A. L. Sasu, B. SASU, Perron conditions for pointwise and global exponential dichotomy of linear skew-product flows, accepted for publication in Integral Equations Operator Theory.

[18] M. Megan, A. L. SAsu, B. SASU, Theorems of Perron type for uniform exponential stability of linear skew-product semiflows, accepted for publication in Dynam. Contin. Discrete Impuls. Systems.

[19] J. VAN NEERVEN, Exponential stability of operators and operator semigroups, J. Funct. Anal. 130 (1995), 293-309.

[20] S. RolewiCZ, On uniform N-equistability, J. Math. Anal. Appl. 115 (1986), 434-441. 